\title{
The First Synthesis of Sessiline
}

\author{
Viktor Ilkei ${ }^{*}$, Kornél Faragó1, Zsuzsanna Sánta², Miklós Dékány², László Hazai' ${ }^{1}$, \\ Csaba Szántay Jr. ${ }^{2}$, Csaba Szántay ${ }^{1}$, György Kalaus ${ }^{1}$ \\ ${ }^{1}$ Department of Organic Chemistry and Technology, Budapest University of Technology and Economics, \\ Budapest, Hungary \\ ${ }^{2}$ Gedeon Richter Plc, Budapest, Hungary \\ Email: ${ }^{*}$ viktor.ilkei@gmail.com
}

Received 9 October 2014; revised 23 November 2014; accepted 9 December 2014

Copyright (C) 2014 by authors and Scientific Research Publishing Inc.

This work is licensed under the Creative Commons Attribution International License (CC BY). http://creativecommons.org/licenses/by/4.0/

(c) (i) Open Access

\section{Abstract}

Sessiline is an alkaloid which was recently isolated from the fruits of Acanthopanax sessiliflorus. The molecule contains two five-membered heterocyclic units joined together by an acylaminocarbinol-ether type bond. Here, we describe the first, simple synthesis of sessiline from 5-hydroxypyrrolidin-2-one and 5-hydroxymethylfurfural, which are prepared from succinimide and furfuryl alcohol, respectively. The coupling reaction takes place on moderate heating under neat conditions.

\section{Keywords}

Sessiline, Acylaminocarbinol, Iminium Ion, Alkaloid Synthesis

\section{Introduction}

Sessiline (1) (Figure 1) was isolated in 2002 from the fruits of Acanthopanax sessiliflorus, a herbaceous plant, which is distributed in East Asia [1]. Its structure was elucidated by spectroscopic methods. The molecule consists of two heterocyclic units joined together by an ether-bond. The alkaloid is found in the plant as a racemate.

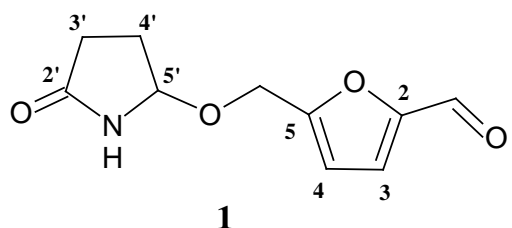

Figure 1. The structure of sessiline (1).

\footnotetext{
${ }^{*}$ Corresponding author.
}

How to cite this paper: Ilkei, V., Faragó, K., Sánta, Z., Dékány, M., Hazai, L., Szántay Jr., C., Szántay, C. and Kalaus, G. (2014) The First Synthesis of Sessiline. International Journal of Organic Chemistry, 4, 309-313. 
It is known that acylaminocarbinols, as well as the iminium ions that arise from them, are electrophilic reagents, which can be utilized in the synthesis of sessiline (1). Taking the above assumption into consideration, we outlined a simple retrosynthetic scheme for 1 (Scheme 1). By disconnecting the ether bond, we obtain two known compounds: 5-hydroxypyrrolidin-2-one (2) and 5-hydroxymethylfurfural (3).

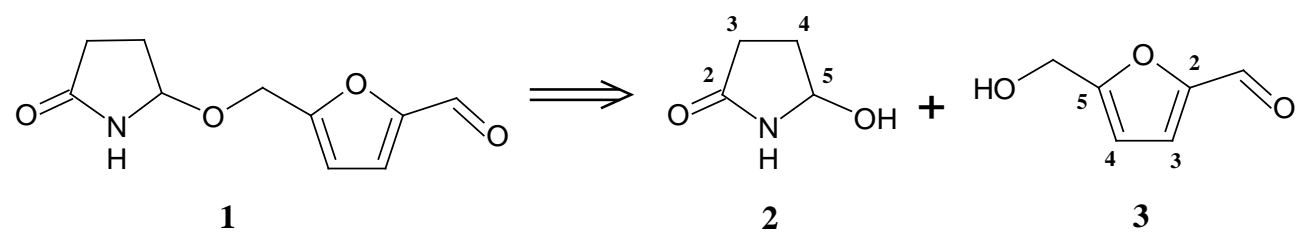

Scheme 1. Retrosynthetic analysis of sessiline (1).

\section{Results and Discussion}

In order to accomplish our goal, we synthesized 5-hydroxypyrrolidin-2-one (2) and 5-hydroxymethylfurfural (3) (Scheme 2). Compound 2 can be prepared from succinimide (4) in two steps. The partial reduction of 4 yields 5-ethoxypyrrolidin-2-one (5) [2], which can be hydrolysed in boiling water to give the amidocarbinol 2 [3].

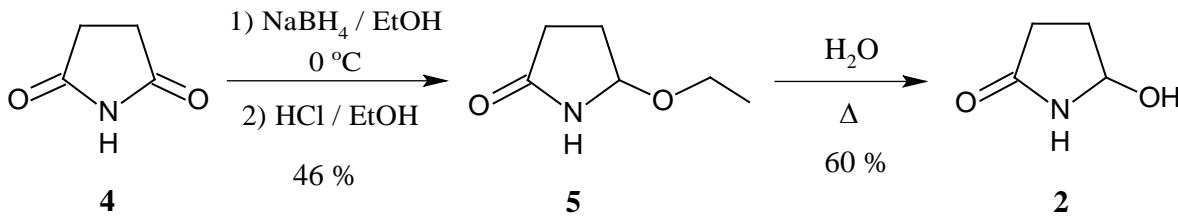

\section{Scheme 2. Synthesis of 5-hydroxypyrrolidin-2-one (2).}

Next, we prepared 5-hydroxymethylfurfural (3) from furfuryl alcohol (6) (Scheme 3). First, 6 was protected by acetylation to give furfuryl acetate (7) [4], which was subjected to Vilsmeier formylation to yield 5-(formyl)furfuryl acetate (8) [5]. Then, $\mathbf{3}$ was obtained by deacetylation [6].

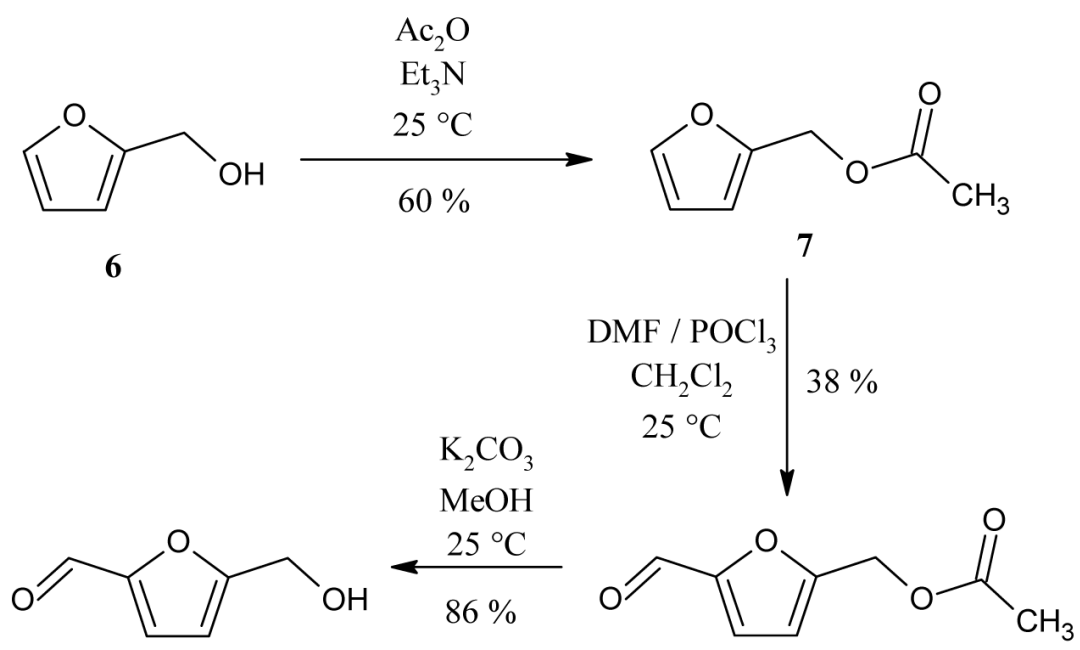

3

8

Scheme 3. Synthesis of 5-hydroxymethylfurfural (3).

Next, we needed to synthesize target molecule $\mathbf{1}$ by coupling $\mathbf{2}$ and $\mathbf{3}$. Although the type of the planned reac- 
tion is known, relatively few examples can be found in the literature. The majority of these transformations were carried out under mild conditions using acid catalysis [7] [8].

In the present case, 2 was allowed to react with an excess of 3 at $60^{\circ} \mathrm{C}$ under neat conditions. The reaction gave sessiline (1) in an acceptable yield (Scheme 4).

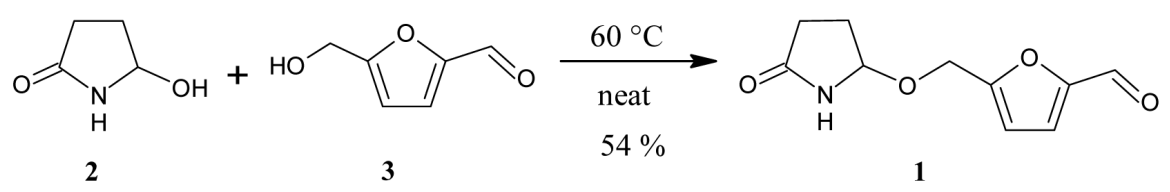

Scheme 4. The synthesis of sessiline (1).

Surprisingly, no acid catalysis or solvent was necessary for the reaction to take place, raising of the temperature proved to be sufficient. In light of our successful synthesis, we proposed a plausible reaction mechanism for the formation of sessiline (1) (Scheme 5). According to our assumption, higher temperatures cause the equilibrium between the amidocarbinol $\mathbf{2}$ and its ionic form 2/a to shift towards the latter, which results in a higher concentration of acyliminium ions in the reaction mixture, thus enabling the reaction to take place.

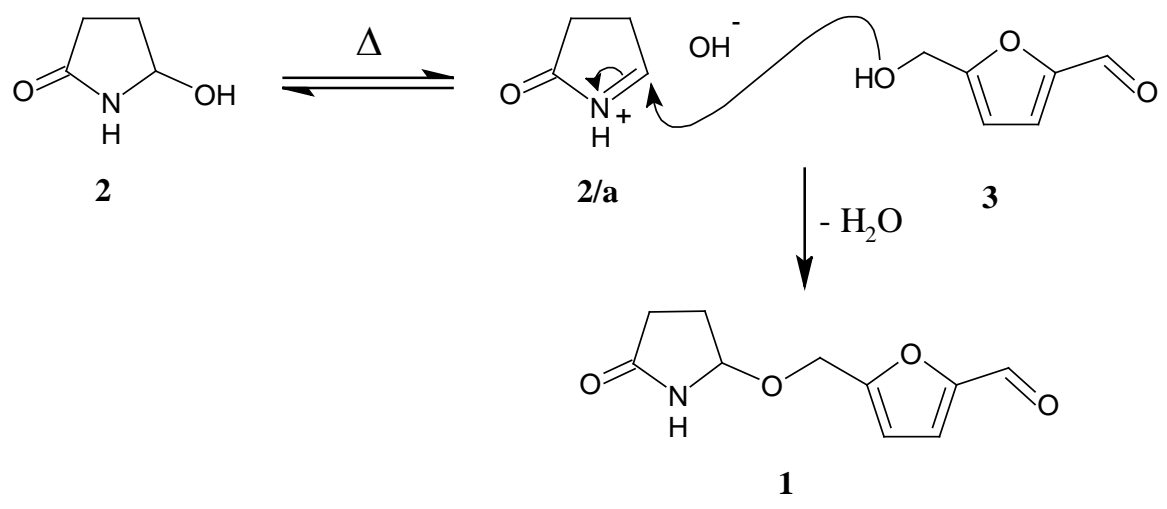

Scheme 5. Proposed reaction mechanism for the formation of sessiline (1).

\section{Experimental}

\subsection{General}

Melting points were measured on a SANYO Gallenkamp apparatus and are uncorrected. IR spectra were recorded on a Bruker FT-IR instrument. ${ }^{1} \mathrm{H}-\mathrm{NMR}$ and ${ }^{13} \mathrm{C}$-NMR measurements were performed on Varian 400 MHz, Varian $500 \mathrm{MHz}$ and Varian $800 \mathrm{MHz}$ spectrometers. Chemical shifts are given on the delta scale as parts per million (ppm) with tetramethylsilane (TMS) $\left({ }^{1} \mathrm{H}\right)$ or dimethylsulfoxide- $d_{6}\left({ }^{13} \mathrm{C}\right)$ as the internal standard $(0.00$ ppm and 39.5 ppm, respectively). MS spectra were recorded on VG-Trio-2 and Finnigan MAT 95SQ instruments using EI or ESI techniques. HRMS analyses were performed on an LTQ FT Ultra (Thermo Fischer Scientific, Bremen, Germany) system. TLC was carried out using Kieselgel $60 \mathrm{~F}_{254}$ (Merck) coated glass plates. Column chromatography was performed using Geduran Si 60 (Merck) silica.

\subsection{Furfuryl Acetate (7)}

To a mixture of $56.6 \mathrm{~g}(577 \mathrm{mmol} ; 50 \mathrm{ml})$ furfuryl alcohol (6) and $12.1 \mathrm{~g}(120 \mathrm{mmol} ; 16.7 \mathrm{ml})$ triethylamine was added $67 \mathrm{~g}(656 \mathrm{mmol} ; 62.4 \mathrm{ml})$ of acetic anhydride dropwise over 15 minutes. The reaction mixture was stirred at ambient temperature for 21 hours, then it was extracted with $3 \times 30 \mathrm{ml}$ water. The organic phase was dried over magnesium sulphate and evaporated under reduced pressure. The crude product was distilled in vacuo (bp. $76^{\circ} \mathrm{C} / 15 \mathrm{mmHg}$; lit.: $67^{\circ} \mathrm{C} / 8 \mathrm{mmHg}$ [4]). $48 \mathrm{~g}(60 \%)$ pure 7 was obtained as a clear liquid. $\mathrm{n}_{\mathrm{d}}$ : 1.4619 (lit.: 1.4603 [4]). IR $v_{\max }\left(\right.$ film, $\mathrm{cm}^{-1}$ ): 1738, 1502, 1437, 1231, 1150, 1079, 1015, 918, 885, 817, 744. 


\subsection{5-(Formyl)Furfuryl Acetate (8)}

A mixture of $97 \mathrm{~g}$ ( $1.33 \mathrm{~mol} ; 103 \mathrm{ml})$ dimethylformamide and $300 \mathrm{ml}$ dichloromethane was cooled to $0^{\circ} \mathrm{C}$. To this mixture $152 \mathrm{~g}$ ( $0.99 \mathrm{~mol} ; 91 \mathrm{ml})$ phosphoryl chloride was added dropwise at $0^{\circ} \mathrm{C}$ over 5 minutes. The reaction mixture was stirred at $0^{\circ} \mathrm{C}$ for 1 hour, then $48 \mathrm{~g}(0.343 \mathrm{~mol} ; 43 \mathrm{ml})$ furfuryl acetate (7) was added dropwise over 5 minutes. The reaction mixture was stirred at ambient temperature for 22 hours, after which it was neutralised with $15 \%$ sodium carbonate solution. The precipitate was filtered and washed with $100 \mathrm{ml}$ dichloromethane. The aqueous-organic mixture was extracted with $10 \times 100 \mathrm{ml}$ dichloromethane, the organic phase was dried over magnesium sulphate and evaporated under reduced pressure. The crude product was crystallized from ether, after which $22.7 \mathrm{~g}$ (39\%) of pure 8 was obtained as colourless crystals. $\mathrm{R}_{\mathrm{f}} 0.81$ (ethyl acetate:hexane = 2:1). Mp.: $55^{\circ} \mathrm{C}-56^{\circ} \mathrm{C}$ (lit.: $55^{\circ} \mathrm{C}-56^{\circ} \mathrm{C}$ [5]). IR $v_{\max }\left(\mathrm{KBr}, \mathrm{cm}^{-1}\right): 3122,2943,2833,1740,1675,1588,1523$, 1437, 1403, 1366, 1273, 1221, 1022. ${ }^{1} \mathrm{H}$ NMR (800 MHz, DMSO-d $\left.d_{6}\right) \delta_{\mathrm{H}} 2.08$ (s, 3H, Ac); $5.15\left(s, 2 \mathrm{H}, \mathrm{CH}_{2} \mathrm{O}\right)$; $6.81(d, 1 \mathrm{H}, J=3.5 \mathrm{~Hz}, \mathrm{H}-4) ; 7.53(d, 1 \mathrm{H}, J=3.5 \mathrm{~Hz}, \mathrm{H}-3) ; 9.60$ (s, $1 \mathrm{H}, \mathrm{CH}=\mathrm{O})$. MS(EI): $168\left(\mathrm{C}_{8} \mathrm{H}_{8} \mathrm{O}_{4}\right)$. EI-MS (rel. int.\%): 168(1); 126(100); 109(22); 97(7); 79(30); 53(9); 44(22); 43(28).

\subsection{5-Hydroxymethylfurfural (3)}

To $2.60 \mathrm{~g}$ (15.5 mmol) 5-(formyl)furfuryl acetate (8) dissolved in $20 \mathrm{ml}$ methanol, $0.330 \mathrm{~g}$ (2.39 mmol) potassium carbonate was added. The reaction mixture was strirred at ambient temperature for 1 hour, after which $15 \mathrm{ml}$ water was added and the mixture was extracted with $10 \times 15 \mathrm{ml}$ dichloromethane. The organic phase was dried over magnesium sulphate and evaporated under reduced pressure. The crude product was subjected to column chromatography using a mixture of ethyl acetate:hexane $=2: 1$ as the eluent. $1.671 \mathrm{~g} \mathrm{(86 \% )}$ of pure 3 was obtained as a yellow liquid, which crystallized on cooling. $\mathrm{R}_{\mathrm{f}} 0.55$ (ethyl acetate:hexane $=2: 1$ ). Mp.: $28^{\circ} \mathrm{C}-32^{\circ} \mathrm{C}$ (lit.: $31^{\circ} \mathrm{C}-32^{\circ} \mathrm{C}$ [6]). IR $v_{\max }\left(\mathrm{KBr}, \mathrm{cm}^{-1}\right)$ : 3405, 3123, 2926, 2851, 1675, 1523, 1397, 1370, $1334,1280,1192,1023 .{ }^{1} \mathrm{H}$ NMR $\left(800 \mathrm{MHz}, \mathrm{DMSO}_{6}\right) \delta_{\mathrm{H}} 4.51(d, 2 \mathrm{H}, J=6.0 \mathrm{~Hz}, \mathrm{CHO}) ; 5.59(t, 1 \mathrm{H}, J=6.0$ $\mathrm{Hz}, \mathrm{OH}) ; 6.61(d, 1 \mathrm{H}, J=3.5 \mathrm{~Hz}, \mathrm{H}-4) ; 7.50(d, 1 \mathrm{H}, J=3.5 \mathrm{~Hz}, \mathrm{H}-3) ; 9.55$ (s, 1H, CH=O). MS(EI): 126 $\left(\mathrm{C}_{6} \mathrm{H}_{6} \mathrm{O}_{3}\right)$. EI-MS (rel. int.\%): 126(96); 109(10); 97(100); 81(4); 69(24); 53(9); 41(50); 39(22).

\subsection{5-Ethoxypyrrolidin-2-0ne (5)}

A solution of $7.156 \mathrm{~g}$ (72.22 mmol) succinimide (4) in $300 \mathrm{ml}$ ethanol was cooled to $0^{\circ} \mathrm{C}$. To this solution $4.00 \mathrm{~g}$ (105.74 mmol) sodium borohydride was added in one portion. The solution was stirred at $0^{\circ} \mathrm{C}$ for 4 hours, during which time every 15 minutes 5 drops of $2 \mathrm{M}$ ethanolic hydrogen chloride solution were added. Then the reaction mixture was acidified to $\mathrm{pH}=3$ with $2 \mathrm{M}$ ethanolic hydrogen chloride solution over 30 minutes, after which it was stirred at $5^{\circ} \mathrm{C}$ for 45 minutes. Then the reaction mixture was neutralised $(\mathrm{pH}=7)$ with $5 \%$ ethanolic potassium hydroxide solution and evaporated to dryness under reduced pressure. The remaining syrupy solid was suspended in $80 \mathrm{ml}$ chloroform, filtered, and the precipitate washed with $3 \times 20 \mathrm{ml}$ chloroform. The filtrate was evaporated under reduced pressure, and the remaining colourless oil was dissolved in $80 \mathrm{ml}$ dichloromethane and washed with $3 \times 10 \mathrm{ml}$ water. The aqueous phase was extracted with $6 \times 20 \mathrm{ml}$ dichloromethane, then the organic phases were unified, dried over magnesium sulphate and evaporated under reduced pressure. The remaining colourless oil crystallized on standing, after which $4.327 \mathrm{~g} \mathrm{(46 \% )}$ pure 5 was obtained as colourless crystals. $\mathrm{R}_{\mathrm{f}} 0.67$ (acetone). Mp.: $51^{\circ} \mathrm{C}-53^{\circ} \mathrm{C}$ (lit.: $48^{\circ} \mathrm{C}-53^{\circ} \mathrm{C}$ [2]). $\mathrm{IR} v_{\max }\left(\mathrm{KBr}, \mathrm{cm}^{-1}\right)$ : 3200, 2978, 1707, 1689, 1668, 1457, 1282, 1250, 1067, 986. ${ }^{1} \mathrm{H}$ NMR (400 MHz, DMSO-d $\left.d_{6}\right) \delta_{\mathrm{H}} 1.10\left(t, 3 \mathrm{H}, J=7.0 \mathrm{~Hz}, \mathrm{CH}_{3}\right) ; 1.78-$ $1.89\left(m, 1 \mathrm{H}, \mathrm{H}_{\mathrm{x}}-4\right)$; 1.95 - $2.05\left(m, 1 \mathrm{H}, \mathrm{H}_{\mathrm{x}}-3\right)$; 2.11 - $2.30\left(m, 2 \mathrm{H}, \mathrm{H}_{\mathrm{y}}-4, \mathrm{H}_{\mathrm{y}}-3\right)$; 3.27-3.35 (m, $\left.1 \mathrm{H}, \mathrm{OCH}_{2 \mathrm{x}}\right)$; $3.44-$ $3.54\left(m, 1 \mathrm{H}, \mathrm{OCH}_{2 \mathrm{y}}\right) ; 4.83-4.89(m, 1 \mathrm{H}, \mathrm{H}-5) ; 8.62(\mathrm{~s}, 1 \mathrm{H}, \mathrm{NH}) .{ }^{13} \mathrm{C}$ NMR (100 MHz, DMSO-d $\left.d_{6}\right) \delta_{\mathrm{C}} 15.1$ $\left(\mathrm{CH}_{3}\right) ; 27.7$ (C-4); 28.1 (C-3); $61.6\left(\mathrm{OCH}_{2}\right) ; 85.0$ (C-5); 177.4 (CON). MS(ESI): $130\left(\mathrm{C}_{6} \mathrm{H}_{12} \mathrm{NO}_{2}\right)$. ESI-MS-MS (cid = 35) (rel. int.\%): 84(100).

\subsection{5-Hydroxypyrrolidin-2-One (2)}

$2.0 \mathrm{~g}$ (15.5 mmol) 5-ethoxypyrrolidin-2-one (5) was dissolved in $25 \mathrm{ml}$ water and the solution was refluxed for 3 hours. Then the water was evaporated under reduced pressure. The remaining oil was triturated with ethyl acetate, from which a white solid crystallized on cooling. The solid was filtered and recrystallized from acetone. $0.89 \mathrm{~g}(60 \%)$ pure 2 was obtained as colourless crystals. $\mathrm{R}_{\mathrm{f}} 0.19$ (acetone:hexane $=2: 1$ ). Mp.: $94^{\circ} \mathrm{C}-96^{\circ} \mathrm{C}$ (lit.: $90^{\circ} \mathrm{C}$ [3]). IR $v_{\max }\left(\mathrm{KBr}, \mathrm{cm}^{-1}\right): 3254,2996,2962,1668,1475,1415,1323,1271,1166,1101,1070,1016 .{ }^{1} \mathrm{H}$ 
NMR (400 MHz, DMSO- $\left.d_{6}\right) \delta_{\mathrm{H}} 1.66-1.75\left(m, 1 \mathrm{H}, \mathrm{H}_{\mathrm{x}}-4\right) ; 1.94-2.03\left(m, 1 \mathrm{H}, \mathrm{H}_{\mathrm{x}}-3\right)$; $2.14-2.24\left(m, 1 \mathrm{H}, \mathrm{H}_{\mathrm{y}}-4\right)$; 2.23 - $2.33\left(m, 1 \mathrm{H}, \mathrm{H}_{\mathrm{y}}-3\right) ; 5.06$ (m, 1H, H-5); $5.70(d, 1 \mathrm{H}, J=6.9 \mathrm{~Hz}, \mathrm{OH}) ; 8.18(\mathrm{~s}, 1 \mathrm{H}, \mathrm{NH}) .{ }^{13} \mathrm{C}$ NMR $(100$ MHz, DMSO- $\left.d_{6}\right) \delta_{\mathrm{C}} 28.4(\mathrm{C}-3)$; $30.3(\mathrm{C}-4) ; 78.5(\mathrm{C}-5) ; 176.7(\mathrm{CON})$. HRMS: $102.05491\left(\mathrm{C}_{4} \mathrm{H}_{8} \mathrm{NO}_{2}\right.$; calc. 102.05496). ESI-MS-MS (cid = 55) (rel. int.\%): 85(100).

\subsection{Sessiline (1)}

$200 \mathrm{mg}$ (1.98 mmol) 5-hydroxypyrrolidin-2-one (2) and $800 \mathrm{mg}$ (6.34 mmol) 5-hydroxymethylfurfural (3) were stirred at $60^{\circ} \mathrm{C}$ under neat conditions for 1 hour. Then the reaction mixture was diluted with 1 - $2 \mathrm{ml}$ dichloromethane, filtered, the solid washed with $3 \times 2 \mathrm{ml}$ dichloromethane, and air-dried with suction. $225 \mathrm{mg}$ (54\%) pure 1 was obtained as colourless crystals. $\mathrm{R}_{\mathrm{f}} 0.47$ (acetone:hexane $=2: 1$ ). Mp.: $169^{\circ} \mathrm{C}-172^{\circ} \mathrm{C}$ (lit.: $171^{\circ} \mathrm{C}$ $172^{\circ} \mathrm{C}$ [1]). IR $v_{\max }\left(\mathrm{KBr}, \mathrm{cm}^{-1}\right)$ : 3321, 3176, 3117, 2965, 2896, 2863, 2790, 2763, 1772, 1709, 1666, 1531, 1463, 1412, 1389, 1338, 1284, 1276, 1262, 1250, 1208, 1201, 1177, 1096, 1062, 1030, 1007. ${ }^{1} \mathrm{H}$ NMR (400 MHz, DMSO- $\left.d_{6}\right) \delta_{\mathrm{H}} 1.86-1.95\left(m, 1 \mathrm{H}, \mathrm{H}_{\mathrm{x}}-4^{\prime}\right) ; 2.01-2.10\left(m, 1 \mathrm{H}, \mathrm{H}_{\mathrm{x}}-3^{\prime}\right) ; 2.16-2.34\left(\mathrm{~m}, 2 \mathrm{H}, \mathrm{H}_{\mathrm{y}}-4^{\prime}, \mathrm{H}_{\mathrm{y}}-3^{\prime}\right) ; 4.49+$ $4.58\left(A B, 2 \times 1 \mathrm{H}, J_{g e m}=13.2 \mathrm{~Hz}, \mathrm{CHO}\right) ; 5.02(m, 1 \mathrm{H}, \mathrm{H}-5$ '); $6.73(d, 1 \mathrm{H}, J=3.4 \mathrm{~Hz}, \mathrm{H}-4) ; 7.51(d, 1 \mathrm{H}, J=3.4$ $\mathrm{Hz}, \mathrm{H}-3) ; 8.80$ (s, 1H, NH); $9.58(\mathrm{~s}, 1 \mathrm{H}, \mathrm{CH}=\mathrm{O}) .{ }^{13} \mathrm{C}$ NMR (100 MHz, DMSO-d $\left.d_{6}\right) \delta_{\mathrm{C}} 27.5$ (C-4'); 27.9 (C-3'); $60.3\left(\mathrm{CH}_{2} \mathrm{O}\right) ; 85.2$ (C-5); 111.8 (C-4); 123.9 (C-3); 152.2 (C-2); 157.7 (C-5); $177.6(\mathrm{CON}) ; 178.3(\mathrm{CH}=\mathrm{O})$. HRMS: $210.07605\left(\mathrm{C}_{10} \mathrm{H}_{12} \mathrm{NO}_{4}\right.$; calc. 210.07608). ESI-MS-MS (cid=65) (rel. int.\%): 192(100); 164(8); 126(2); 109(4).

\section{Acknowledgements}

The authors are grateful to Gedeon Richter Plc for the financial support.

\section{References}

[1] Lee, S., Ji, J., Shin, K.H. and Kim, B.-K. (2002) Sessiline, A New Nitrogenous Compound from the Fruits of Acanthopanax sessiliflorus. Planta Medica, 68, 939-941. http://dx.doi.org/10.1055/s-2002-34925

[2] Hubert, J.C., Wijnberg, J.B.P.A. and Speckamp, W.N. (1975) $\mathrm{NaBH}_{4}$ Reduction of Cyclic Imides. Tetrahedron, 31, 1437-1441. http://dx.doi.org/10.1016/0040-4020(75)87076-1

[3] Cue Jr., B.W. and Chamberlain, N. (1979) An Improved Method for the Preparation of 5-Hydroxy-2-Pyrrolidone. Organic Preparations and Procedures International, 11, 285-286. http://dx.doi.org/10.1080/00304947909355413

[4] Renvall, I. and Mattila, T. (1977) Esterification of Furfuryl Alcohol and Its Derivatives. US Patent No. 4008256.

[5] Mehner, A., Montero, A.L., Martinez, R. and Spange, S. (2007) Synthesis of 5-Acetoxymethyl- and 5-Hydroxymethyl2-Vinylfuran. Molecules, 12, 634-640. http://dx.doi.org/10.3390/12030634

[6] Schinzer, D., Bourguet, E., Ducki, S. (2004) Synthesis of Furano-Epothilone D. Chemistry-A European Journal, 10, 3217-3224. http://dx.doi.org/10.1002/chem.200400125

[7] Toja, E., Gorini, C., Zirotti, C., Barzaghi, F. and Galliani, G. (1991) Amnesia-Reversal Activity of a Series of 5-Alkoxy1-Arylsulfonyl-2-Pyrrolidinones. European Journal of Medicinal Chemistry, 26, 403-413. http://dx.doi.org/10.1016/0223-5234(91)90101-R

[8] Toja, E., Gorini, C., Zirotti, C., Barzaghi, F. and Galliani, G. (1991) Amnesia-Reversal Activity of a Series of 5-Alkoxy1-Arylcarbonyl-2-Pyrrolidinones and 5-Alkoxy-1-Arylmethyl-2-Pyrrolidinones. European Journal of Medicinal Chemistry, 26, 415-422. http://dx.doi.org/10.1016/0223-5234(91)90102-S 
Scientific Research Publishing (SCIRP) is one of the largest Open Access journal publishers. It is currently publishing more than 200 open access, online, peer-reviewed journals covering a wide range of academic disciplines. SCIRP serves the worldwide academic communities and contributes to the progress and application of science with its publication.

Other selected journals from SCIRP are listed as below. Submit your manuscript to us via either submit@scirp.org or Online Submission Portal.
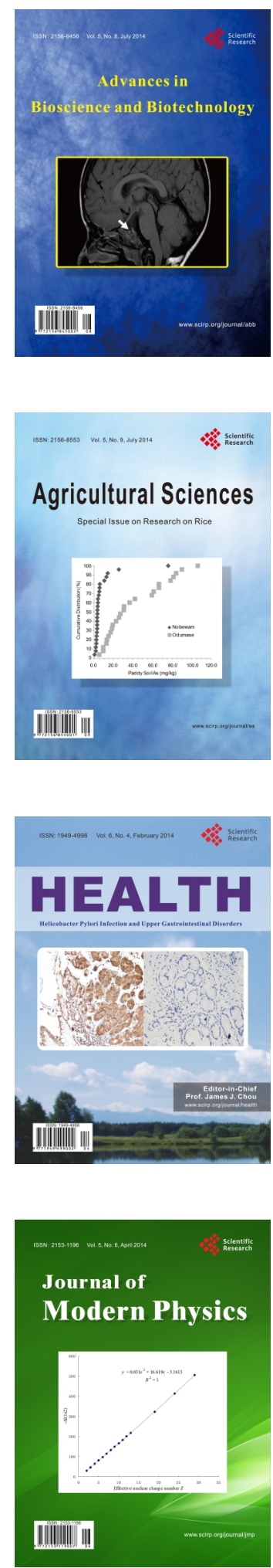
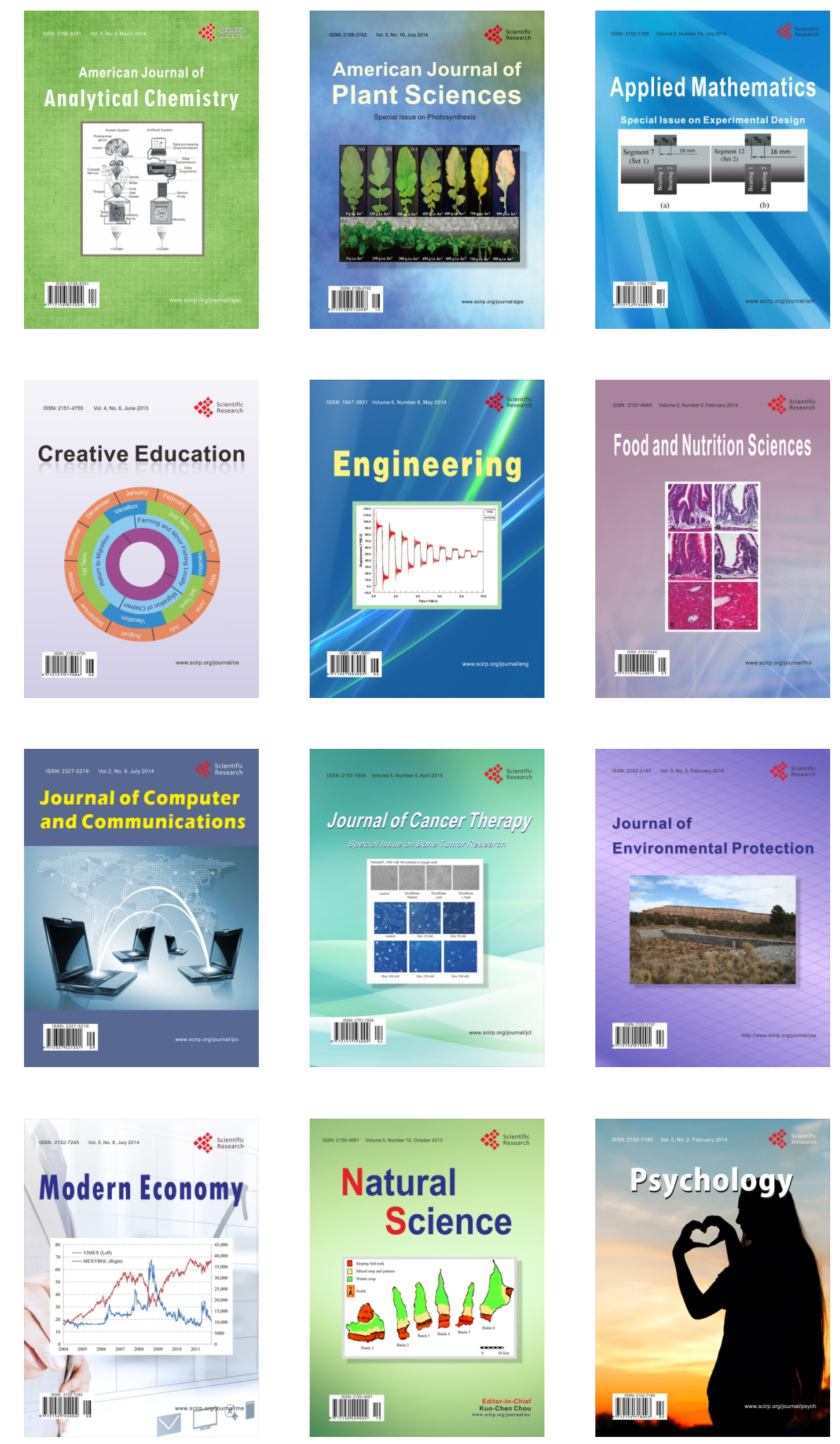